\title{
Ultrasound study of layer-specific strain and perfusion of left ventricular myocardium in patients with dilated cardiomyopathy
}

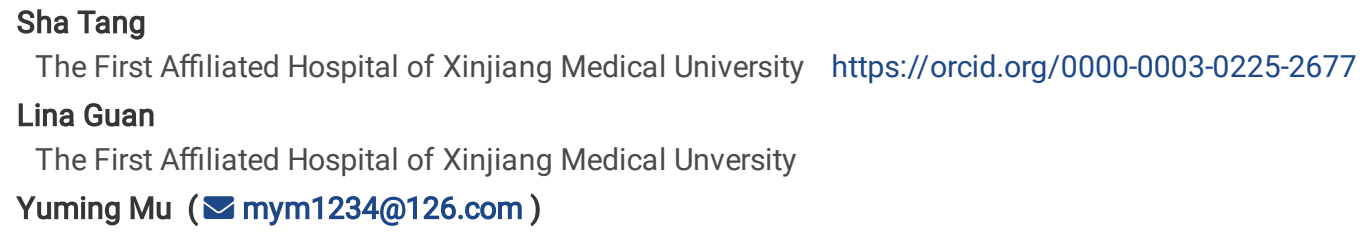

\section{Research}

Keywords: Dilated cardiomyopathy, Two-dimensional speckle tracking, Myocardial contrast echocardiography, Myocardial microcirculation perfusion

Posted Date: July 6th, 2021

DOI: https://doi.org/10.21203/rs.3.rs-153961/v3

License: (c) (i) This work is licensed under a Creative Commons Attribution 4.0 International License. Read Full License 


\section{Abstract \\ Purpose}

To investigate the changes in deformation and myocardial microcirculation perfusion of left ventricular three-layer myocardium in patients with dilated cardiomyopathy (DCM) by using speckle tracking imaging (STI) and myocardial contrast echocardiography (MCE).

\section{Methods}

Twenty-four patients with DCM and 19 healthy controls were selected. Two-dimensional and MCE dynamic images of apical four-chamber, twochamber, and three-chamber sections and left ventricular mitral valve, papillary muscle and apex sections were collected. The peak values of longitudinal strain (LS), circumferential strain (CS), cross-sectional area of a microvessel (A) and average myocardial microvascular lesion ( $\beta$ ) were obtained by Qlab 10.8 workstation values, and myocardial blood flow (MBF) was calculated with $A \times \beta$ to evaluate the deformation and coronary microvascular perfusion of left ventricular three-layer myocardium.

\section{Results}

The brain natriuretic peptide (BNP), left ventricular mass index (LVMI), left ventricular end-diastolic diameter (LVEDD), left ventricular end-systolic diameter (LVEDS), left ventricular end-diastolic volume (LVEDV), left ventricular end-systolic volume (LVESV), left atrial volume index (LAVI), E peak in early diastolic period/A peak velocity in late diastolic period (E/A) and average E/e' in the DCM group were higher than those in the control group $(P<0.05)$; left ventricular ejection fraction (LVEF), left ventricular fractional shortening (FS) rate, stroke volume (SV), cardiac output $(\mathrm{CO})$, cardiac index $(\mathrm{Cl})$, A peak, and the e' and a' velocities of both the lateral wall and interventricular septum were smaller than those in the control group $(P<0.05)$. The LS, CS, A, $\beta$, and $A \times \beta$ of the DCM group were all lower than those of the control group, The time to peak and the cardiac cycle required to reach the peak were longer than those in the control group $(P<0.05)$. The pattern of myocardial strain and perfusion among myocardial layers was subendocardial > middle > subepicardial. The correlation coefficients of LS with $A, \beta$, and $A \times \beta$ were $-0.500,-0.279$ and -0.190 , respectively, and the correlation coefficients of CS with $A, \beta$, and $A \times \beta$ were $-0.383,-0.255$ and -0.208 , respectively.

\section{Conclusions}

The deformation of the three-layer myocardium and coronary microcirculation perfusion in DCM patients were diffusely damaged from the endocardium to the epicardium, layer by layer. The longitudinal function of the left ventricular myocardium was closely related to changes in myocardial microcirculation perfusion. Echocardiography is helpful to evaluate myocardial blood flow and myocardial ischemia in patients with DCM.

\section{Background}

Dilated cardiomyopathy (DCM) is a primary cardiomyopathy without abnormal hemodynamic load (such as aortic stenosis, hypertension or mitral regurgitation) and coronary artery disease, characterized by varying degrees of dilation of the left or both ventricles and myocardial dysfunction ${ }^{[1]}$, whose prevalence is approximately $1: 250^{[2]}$. DCM is an important cause of sudden cardiac death (SCD) and heart failure (HF) ${ }^{[3]}$, which is the main indication for heart transplantation ${ }^{[4]}$. Some studies have shown that in addition to changes in cardiac structure and function, the coronary microvascular system of DCM patients is also directly affected ${ }^{[5]}$; moreover, its dysfunction can cause regional myocardial perfusion, wall motion and metabolic abnormalities of the left ventricle, and this is correlated with cardiac events ${ }^{[6]}$. Therefore, changes in cardiac structure and function and evaluation of the coronary microvascular system in DCM patients are key issues in the process of clinical diagnosis and treatment.

Echocardiography plays a fundamental and important role in the imaging diagnosis of DCM because of its noninvasive, safe, reliable and convenient characteristics. It provides a complete and feasible morphological and functional evaluation of DCM by observing the severity of cardiac anatomy, pathophysiology and hemodynamics ${ }^{[7]}$. Speckle tracking imaging (STI) objectively quantifies myocardial deformation and left ventricular systolic and diastolic function by analyzing the movement of spots on conventional two-dimensional echocardiography. The myocardial strain parameters measured by STI are sensitive indicators of the change in fiber length in the process of ventricular contraction, which can be used to measure the mechanical function and deformation of the left ventricular myocardium in DCM patients and evaluate the abnormality of myocardial function. Myocardial contrast echocardiography (MCE) is a noninvasive imaging technique ${ }^{[8]}$ that uses microbubbles that are completely confined to the coronary capillaries after intravenous administration to simulate erythrocyte rheology, evaluate local myocardial microvascular perfusion and detect abnormal perfusion. It has high spatial and temporal resolution, and it is easy to perform and repeat and free of radioactive contamination. The measured values of MCE and intracoronary Doppler flow in animals and humans have been 
reported to be the same ${ }^{[9]}$ and can detect the capillary blood volume and evaluate the myocardial blood flow (MBF) through its time resolution. MCE has been widely used to evaluate microcirculatory dysfunction of the coronary artery[10].

According to the shape of the myocardial bundle, the left ventricular wall is divided into oblique, annular and longitudinal layers. According to the anatomy of the three-layer muscle band of the myocardium, the left ventricular myocardium can be divided into inner, middle and outer layers. The strain can be measured to reflect the true movement of the myocardium and detect incomplete myocardial injury of the left ventricle in the early stage of the disease ${ }^{[11]}$. The microcirculation of the coronary artery is mainly composed of the anterior artery (diameter $100 \sim 500 \mu \mathrm{m}$ ), arteriole (diameter < $100 \mu \mathrm{m}$ ), capillary and venule. Most arterioles, capillaries and venules run parallel to the longitudinal axis of myocardial cells, which determines the main factors of coronary flow. The arteriole is located in the heart muscle, and its resistance accounts for $55 \%$ of the total resistance of coronary blood volume. The capillaries and veins are mainly volume vessels, accounting for approximately $90 \%$ of the heart blood volume ${ }^{[12]}$. The purpose of this study was to evaluate the changes in left ventricular three-layer myocardial deformation and myocardial microcirculation perfusion in DCM patients by STI and MCE.

\section{Methods}

\subsection{Study population}

We selected 24 DCM patients who were hospitalized in the First Affiliated Hospital of Xinjiang Medical University from March 2019 to October 2019, including 17 males and 7 females, with an average age of $44.08 \pm 8.89$ years and 19 healthy controls, including 13 males and 6 females, with an average age of $41.79 \pm 7.23$ years. The inclusion criteria were left ventricular dysfunction (measured by the modified Simpson's method, ejection fraction $<45 \%)^{[13]}$ and normal coronary angiography. Patients with heart valve disease, congenital heart disease, hypertension, coronary heart disease (acute coronary syndrome, vascular reconstruction history, any coronary artery stenosis $>50 \%$ ), diabetes, thyroid disease, severe arrhythmia and other cardiovascular diseases, chronic kidney disease (> stage 3 nephropathy), systemic or immune system disease history, and poor image quality were excluded.

\subsection{Conventional echocardiography}

All subjects were examined by transthoracic echocardiography with Philips 7C color echocardiography and S5-1 phased array probe (frequency 1-5 MHz), and electrocardiograms were connected synchronously. Left atrial (LA) diameter, left ventricular end-diastolic diameter (LVEDD), left ventricular end-systolic diameter (LVEDS), the thicknesses of the interventricular septum (IVS) and left ventricular posterior wall (LVPWs), fractional shortening (FS), stroke volume (SV), and cardiac output (CO) were measured by M-mode ultrasound ${ }^{[14]}$; left ventricular end-diastolic volume (LVEDV), left ventricular end-systolic volume (LVESV), left ventricular ejection fraction (LVEF), and left atrial volume (LAV) were measured by the modified Simpson's method. The left ventricular mass (LVM) was calculated by the Devereux formula ${ }^{[15]}$. The cardiac index (CI), left ventricular mass index (LVMI) and left atrial volume index (LAVI) were standardized by body surface area (BSA). The ratio of mitral peak early (E) and late $(A)$ filling velocity (E/A) were measured by pulsed-wave Doppler (PW) imaging of the mitral inflow. PW of the mitral inflow and tissue Doppler imaging (TDI) of the mitral annulus at the septal and lateral points were performed. The average value of the mitral annular velocities $\left(\mathrm{e}^{\prime}\right)$ and the ratio of $E$ and $e^{\prime}\left(E / e^{\prime}\right)$ were calculated ${ }^{[16]}$.

\subsection{D-STE}

Data from three-layer speckle tracking were analyzed offline using dedicated automated software (QLAB10.8 Philips Healthcare, Andover, MA). The strain analysis from the four-chamber, two-chamber, and three-chamber views and the horizontal views of the left ventricular mitral valve orifice, papillary muscle and apex were entered. Next, in 2-DMQ mode, the thickness was set to $0.25 \mathrm{~cm}$, and the inner, middle and outer layers of the abovementioned slices were outlined manually in sequence. The software can automatically track and contour the myocardium frame by frame to form the corresponding region of interest (ROI). After each layer of the myocardium was automatically divided into 18 segments according to different sections and levels, the longitudinal strain (LS) peak value and the circumferential strain (CS) peak value of each segment in the three layers of the left ventricle could be obtained囚Figure $1 \otimes$.

\subsection{MCE}

The infusion channel was opened by the median vein in the left elbow, and $2.4 \mathrm{ml}$ of contrast was administered by slow intravenous injection over a period of $2 \mathrm{~min}$, followed by slow drip irrigation with 5-ml saline infusion. Meanwhile, we carried out continuous dynamic acquisition. During the testing process, attention must be paid to whether the subjects have any adverse reactions, recording the time from the injection of contrast into the left elbow median vein to the beginning of myocardial development with visual observation, observing the apical 4-chamber and 2-chamber heart images, papillary muscle horizontal left ventricular short axis section, and the filling of ultrasound microbubbles in the heart chamber and myocardium. Periodically, ultrasonic emission with high mechanical index (flash) was applied to break microbubbles in the myocardium to observe myocardial reperfusion. At the same time, hemodynamic changes before and after contrast heart rate (HR), systolic 
blood pressure (SBP), diastolic blood pressure (DBP) and resting oxygen saturation and adverse reactions were recorded to evaluate the safety of SonoVue. The acquired dynamic image was played back after inspection, and the results were analyzed by 2 independent cardiac ultrasound doctors. We obtained satisfactory left ventricular and myocardial visualization in all patients. After $8 \sim 40$ (average of 12.23 \pm 6.75 ) s of intravenous injection, the left chamber was visible. In another $5 \sim 8$ cardiac cycles, using a flash with a high mechanical index, ultrasound fragmented the microbubbles after visualization of the left ventricular myocardium, allowing observation of the condition of myocardial perfusion again ${ }^{[17]}$. We used 10.8 software for analysis, entered the ROI mode, selected (sampling volume $2.5-4 \mathrm{~mm}^{2}$ ) the ROIs of each segment of the left ventricle, including the interventricular septum, anterior wall, lateral wall, inferior wall and posterior wall, and selected the gamma variable. The software automatically displayed the fitting curve of myocardial perfusion intensity and time in each layer and segment. After analyzing the curve, the cross-sectional area of a microvessel (A) and average myocardial microvascular lesion ( $\beta$ ) were determined, MBF was calculated with $A \times \beta$, and Time to peak reflects the time required for myocardial contrast imaging to reach the peak intensity, the cardiac cycle required for myocardial contrast imaging to reach peak intensity is obtained by heart rate standardization: cardiac cycle $=$ time to peak / 60 / heart rate)(Figure 2).

\subsection{Statistical analysis}

JMP13.0 (SAS Institute, Inc.) software was used for statistical analysis. Numerical variables are presented as the mean \pm standard deviation (SD). A $t$ test was used for comparisons between two groups. $T^{\prime}$ test was used when variance was uneven. Analysis of variance was used for multigroup comparisons, and the rank sum test was used for nonpositive data. Absolute number and constituent ratio were used for statistical description of qualitative data, and the Chi-squared test was used for statistical inference. The correlation was analyzed by rank correlation of two variables. $P<.05$ was considered indicative of statistical significance.

\section{Results}

\subsection{General information and conventional echocardiography}

There was no significant difference in age, HR, SBP, DBP, BSA, creatinine, C-reactive protein, IVS, LVPW, or E peak between the two groups. BNP in the DCM group was higher than that in the control group $(P<0.05)$. LVMI, LVEDD, LVEDS, LVEDV, LVESV, LA, LAVI, E/A, and average E/e $\rrbracket^{\text {in }}$ the DCM group were greater and LVEF, FS, SV, CO, Cl, a peak A, interval a' and $\mathrm{e}^{\square}$, sidewall $\mathrm{a}^{\prime}$ and $\mathrm{e}^{\natural}$ were lower than those of the control group, and the differences were statistically significant (Table 1).

\subsection{Comparison of layered strain and layered perfusion}

LSendo, LSmid, LSepi, CSendo, CSmid, CSepi, Aendo, Amid, Aepi, ßendo, Bmid, ßepi, A×ßendo, A×ßmid, and A×ßepi in the DCM group were lower than those in the control group. The time to peak and the cardiac cycle required to reach the peak were longer than those in the control group. The layered strain of the two groups of myocardium showed the trend of LSendo $>$ LSmid $>$ LSepi and CSendo $>$ CSmid $>$ CSepi. The layered myocardial perfusion in both groups also showed the trend of Aendo $>$ Amid $>$ Aepi, $\beta$ endo $>\beta$ mid $>\beta e p i, A \times \beta e n d o>A \times \beta m i d>A \times \beta e p i$. There were significant differences in $A, \beta$, and $A \times \beta$ between the inner layer and the other two layers (Table 2).

\subsection{Correlation between layered strain and layered perfusion}

The correlation coefficient between LSendo and Aendo was - 0.500 , between LSmid and Amid was -0.279 , between LSepi and Aepi was -0.190, between CSendo and Aendo was -0.383 , between CSmid and Amid was -0.255 , and between CSepi and Aepi was -0.208 . The difference was statistically significant (Table 3, Figure 3).

\section{Discussion}

DCM is one of the most common causes of heart failure. It is the most common primary myocardial disease and is characterized by ventricular dilation and myocardial dysfunction without hypertension, valvular disease, congenital heart disease or ischemic heart disease. The pathological basis involves extensive myocardial degeneration, atrophy and fibrosis, and myocardial interstitial fibrosis is the most common form. Its main characteristics are left ventricular enlargement, diffuse reduction in ventricular wall motion, and decreased systolic and diastolic functions. In patients with suspected heart failure or left ventricular dysfunction, echocardiography is the most important means to determine the diagnosis of dilated cardiomyopathy by assessing the presence and severity of left ventricular dilation and dysfunction. Some studies suggest that left ventricular structure and myocardial systolic and/or diastolic function also change with the complex geometric changes in the left ventricular in the development of congestive heart failure in patients with DCM. In the absence of abnormal load or coronary artery disease, the reduction in EF and left ventricular diastolic function can preliminarily diagnose DCM ${ }^{[18]}$. In this study, LVEDD, LVEDS, LVMI, LVEDV, and LVESV were increased, and LVEF, FS, SV, CO, and CI were decreased in the DCM group, which can be used to evaluate the severity of left ventricular dilation and left ventricular systolic function decrease. LA, LAVI, average E/e', and E/A increased, whereas septal e' and lateral e' decreased, suggesting that DCM patients had a certain degree of diastolic dysfunction with decreased left ventricular systolic function. It is believed that there is a disorder and 
degeneration of myocardial fibers in DCM. Collagen fiber hyperplasia and glycogen content reduction were observed in myocardial tissue, which caused patients to have myocardial contractile weakness and significantly reduced left ventricular function, similar to the results of previous studies $^{[19]}$.

According to the arrangement of the cardiac muscle bundle, the left ventricle is composed of three myocardial fiber layers: superficial obliquely directed fibers, middle circumferentially directed fibers and a deep inner layer with longitudinal fibers. The three layers of myocardium account for $25 \%, 53-59 \%$, and $20 \%$ of the ventricular wall thickness, respectively ${ }^{[20]}$. The long axis of the subendocardial fibers is more or less at right angles to the long axis of the fibers forming the subepicardial layer. The endocardial and epicardial myocardial bundles originate from the base, surround the apex of the heart, and then return to the basal area to form a spiral structure. Longitudinal strain mainly reflects longitudinal myocardial fiber function under the endocardium, while circumferential strain mainly reflects annular myocardial fiber function in the middle layer ${ }^{[21]}$. Studies have shown that longitudinal arrangement (up to 70\%) is the most common type of left ventricular myocardial fiber. Longitudinal fibers have an important contribution to overall ventricular function. The longitudinal peak strain is significantly related to myocardial fibrosis ${ }^{[22,23,24]}$, and the longitudinal function is closely related to hemodynamic changes rather than changes in short axis performance ${ }^{25]}$. However, the results of layered speckle tracking analysis showed that the LS and CS of the three layers in patients with DCM were significantly lower than those in the normal group, showing LSendo > LSmid > LSepi, CSendo > CSmid > CSepi, which indicating that the overall left ventricular function was impaired, longitudinal and axial function were reduced, and the degree and direction of myocardial injury in each layer was different. Both LS and CS were decreased layer by layer from the endocardium to the epicardium. The strain represents the deformation of myocardial fiber relative to the original size ${ }^{[26]}$, whose production is not isolated but integral. The motion of the left ventricular wall depends on the synergistic effect between longitudinal and circumferential muscle bundles from all directions. DCM myocardium changes due to loss of cardiomyocytes, "slippage" of cardiomyocytes in the wall, interstitial fibrosis with residual cardiomyocyte hypertrophy, etc. The myocardial structure changes due to fiber disorder and/or an increase in the proportion of the longitudinal myocardial tract ${ }^{[27]}$, which causes the left ventricular cavity in DCM patients to be enlarged, the left ventricular mass and volume to be increased, the myocardial rotation angle to be reduced, the longitudinal and circular motion and deformation ability of the three left ventricular layers to be decreased, and the peak strain to be decreased ${ }^{[28]}$. Due to the difference in tension caused by the different ratio of curvature of the left ventricular endocardium and epicardium, the degree of subendocardial myocardial fibrosis in DCM patients is greater than that of the middle and subepicardial myocardium ${ }^{[29]}$, which resulted in the decrease in LS and CS from endocardium to epicardium. Although the LS and CS of the three layers were significantly reduced, compared with the control group, the trend of layer-by-layer reduction from the epicardium to the endocardium was maintained. It is believed that the effect of myocardial fibrosis on the structural and functional changes in each layer of myocardium is balanced and diffuse ${ }^{[30]}$.

Although DCM is considered a nonischemic myocardial disease, patients can also experience diffuse changes in MBF in the early stages of the disease. MBF injury is multifactorial ${ }^{[31]}$, and regardless of the pathophysiology, damage to MBF in DCM may lead to myocardial ischemia and progressive deterioration of myocardial function. Most of our knowledge about the regulation of coronary circulation and the characteristics of coronary perfusion are based on measurements obtained from epicardial coronary artery and tissue perfusion measurements. However, the measurements obtained from the epicardial coronary artery may mask the true blood flow of myocardial vessels due to its main function ${ }^{[32]}$.

The coronary artery system is composed of three parts with different functions: the proximal segment is represented by the epicardial coronary artery, which has the volume function; the middle segment is represented by the anterior artery, which has the function of maintaining the pressure at the beginning of the small artery within a narrow range; and the distal segment is represented by the intramural small artery, which has the function of matching myocardial blood supply and oxygen consumption ${ }^{[33]}$. The latter two constitute the coronary microcirculation and are the main factors determining coronary blood flow. Among them, the distal small artery is the main part of MBF metabolic regulation, and $90 \%$ of the myocardial blood volume is located in the capillaries, which leads to a gradient increase in coronary perfusion in the myocardium and more subendocardial perfusion. Therefore, the research results show that $A, \beta$, and $A \times \beta$ in the two groups decreased layer by layer from the endocardium to the extracardiac region. Because of the specific and complex nonspecific histopathological features of DCM, its changes involve all myocardial components, including cardiomyocytes, interstitium, small blood vessels and endocardium ${ }^{[34]}$. At the same time, patients with DCM in the end stage show spherical dilation, wall stress increases, and oxygen demand increases resulting in the myocardium undergoing recurrent ischemia. The corresponding increased metabolic demand cannot be met by increasing the blood vessel density, which further aggravates myocardial ischemia, decreased left ventricular function and abnormal MDF ${ }^{[35,36]}$. Furthermore, the branches of the coronary artery in the epicardium often penetrate into the myocardial layer vertically and generate branches in the endocardial layer. To provide effective oxygen delivery, the ratio of capillary and myocardial fibers is 1:1. Most of the distal microarteries and capillaries pass through the length of myocardial cells; their direction is parallel to the direction of the myocardial fiber, and each capillary has a region responsible for its nutrition ${ }^{[37]}$. The endocardium is the farthest layer of the extracorporeal coronary artery blood flow. During the process of contraction and relaxation, the pressure and blood flow of the endocardium will fluctuate violently. Alterations in coronary microcirculation are easily observed in the early stages of disease ${ }^{[38]}$. Therefore, although the myocardial blood volume, blood flow velocity and blood flow of three layers of left ventricular myocardium in patients with DCM were all damaged, compared with the control group, the layer-by-layer decreasing trend from the 
epicardium to the endocardium was maintained. The changes in the myocardial microcirculation perfusion function in DCM patients were diffuse.

In patients with DCM, the myocardial function is further impaired due to abnormal coronary endothelial function (including resistance and conduction vessels). The blood flow velocity in the coronary artery is related to the diameter of the blood vessel, and the diameter of the capillaries is very narrow. The rheology of MCE microbubbles is almost the same as that of erythrocytes in microvascular system, and it can better evaluate the hemodynamics of coronary capillaries. The results of this study show that the peak time of myocardial perfusion in patients with DCM was delayed in addition to the damage of myocardial blood volume, blood velocity and blood flow. It was considered that in the process of MCE examination in patients with DCM, about 2-3 cardiac cycles were observed more than that in normal subjects. Studies have shown that, no matter what the reason, coronary microcirculation injury may lead to repeated myocardial ischemia, further aggravate ventricular remodeling and clinical symptoms ${ }^{[39]}$. Coronary microcirculation was indispensable in the pathophysiology of both ischemic and non-ischemic cardiomyopathy. Even in DCM patients with secondary severe left ventricular dysfunction, the presence of microcirculation abnormalities indicates an increase in morbidity and mortality ${ }^{[40]}$. The changes of MCE data played an important role in the occurrence and development of DCM, and also had important value in the prognosis of patients with DCM. We need to pay attention to the evaluation of myocardial blood flow and myocardial ischemia in patients with $\mathrm{DCM}^{[41]}$.

Correlation analysis results showed that only LSendo and Aendo had a relatively significant correlation. Combined with the above research results, it is considered that left ventricular myocardium is dominated by longitudinally arranged myocardial fibers, which has an important contribution to the overall ventricular function. The fibrosis degree of endocardium in DCM patients is greater than that of the middle and epicardium, although the regional myocardial blood volume, MBF velocity and that MBF of the three-layer myocardium are seriously impaired, and the longitudinal function of the left ventricular myocardium was closely related to the changes in myocardial microcirculation perfusion, the correlation between the endocardial microcirculation perfusion and myocardial strain was obvious. Because the change in myocardial mechanics is a very complex process, the myocardial deformation of each layer depends on the active function of the layer and the passive motion of adjacent layers. The myocardial fiber layers are not isolated but interact with each other. The division of the myocardial layer itself is a kind of rough classification based on research and is not absolute, and it has a one-to-one correspondence with myocardial fibers ${ }^{[42]}$. Although the mechanical and perfusion of left ventricle in DCM patients changed respectively, the correlation between them was weak due to the limitations of the current analysis methods.

\section{Study limitations}

Our study has several limitations: (1) the number of patients in the control group was relatively small; (2) patients with dilated cardiomyopathy in the study did not undergo myocardial biopsy and did not choose other examination methods that are more sensitive to myocardial deformation and perfusion as a reference; (3) there was no clear boundary between the three layers of left ventricular myocardium in the image, and the analysis software used in the study also failed to perform automatic stratification. The choice of the three-layer myocardium is manually selected by the researcher, which may affect the accuracy of the data. (4) Although MCE is a sensitive method for detecting coronary microcirculation disturbance and myocardial viability, its qualitative diagnosis mainly depends on the subjective interpretation of regional perfusion by experienced clinicians. There are few quantitative analysis studies on three-layer myocardial perfusion, and the sample size needs to be expanded for further research.

\section{Conclusion}

In patients with DCM, the longitudinal and circumferential strain of the three layers of left ventricular myocardium were significantly decreased, and the gradient trend from endocardium to endocardium was maintained. The effect of myocardial fibrosis on the structural and functional changes in each layer was balanced and diffuse. The changes in the microcirculation perfusion function of the three layers were also diffuse. LSendo and Aendo showed a significant correlation between left ventricular myocardial strain and microcirculation perfusion, suggesting that the longitudinal function of the left ventricular myocardium is closely related to changes in myocardial microcirculation perfusion. Although the middle and outer myocardial strains were also damaged, there was no significant correlation between them. We need to pay attention to the evaluation of myocardial blood flow and myocardial ischemia in patients with DCM by echocardiography, further improve the level of diagnosis and prognosis, so as to better help clinicians to intervene and manage DCM patients.

\section{Declarations}

\section{Acknowledgements}

This project was supported by a grant from the Program of Autonomous Region (Science and Technology Aid Xinjiang Program, Grant No.2018E02062).ST was responsible for data analysis and writing the manuscript. LG was responsible for data collection. YM was mainly responsible for the experimental design and critical revision of article. 
Funding: This project was supported by a grant from the Program of Autonomous Region (Science and Technology Aid Xinjiang Program, Grant No.2018E02062)

Conflicts of interest/Competing interests: The authors declare that they have no competing interests.

Availability of data and material: The datasets used and/or analysed during the current study are available from the corresponding author on reasonable request.

Code availability: Not applicable.

Authors' contributions: ST was responsible for data analysis and writing the manuscript. LG was responsible for data collection. YM was mainly responsible for the experimental design and critical revision of article.

Ethics approval: This study was approved by the Ethics Committee of the First Affiliated Hospital of Xinjiang Medical University.

Consent to participate: All patients were informed and signed the informed consent form.

Consent for publication: All presentations of case reports have consent for publication.

\section{Abbreviations}

A cross-sectional area of a microvessel

BNP brain natriuretic peptide

BSA body surface area

CI cardiac index

CO cardiac output

CS circumferential strain

DBP diastolic blood pressure

DCM dilated cardiomyopathy

E/A E peak in early diastolic period/A peak velocity in late diastolic period

FS fractional shortening

$\mathrm{HF}$ heart failure

HR heart rate

IVS interventricular septum

LAV left atrial volume

LAVI left atrial volume index

LS longitudinal strain

LVEDD left ventricular end-diastolic diameter

LVEDS left ventricular end-systolic diameter

LVEDV left ventricular end-diastolic volume

LVEF left ventricular ejection fraction

LVESV left ventricular end-systolic volume

LVM left ventricular mass

LVMI left ventricular mass index

LVPWs left ventricular posterior wall

MBF myocardial blood flow

MCE myocardial contrast echocardiography

SBP systolic blood pressure

SCD sudden cardiac death

STI speckle tracking imaging 
SV stroke volume

$\beta$ average myocardial microvascular lesion

\section{References}

1, Maron BJ, Towbin JA, Thiene G, et al (2006) Contemporary definitions and classification of the cardiomyopathies. Circulation 113:1807-1816. https://doi.org/10.1161/CIRCULATIONAHA.106.174287.

2. Hershberger RE, Hedges DJ, Morales A (2013) Dilated cardiomyopathy: the complexity of a diverse genetic architecture. Nat Rev Cardiol 10:531-547. https://doi.org/ 10.1038/nrcardio.2013.105

3. Merlo M, Sinagra G, Carniel E, et al (2013) Poor prognosis of rare sarcomeric gene variants in patients with dilated cardiomyopathy. Clin Transl Sci 6:424-428. https://doi.org/ 10.1111/cts.12116

4. Merlo M, Pivetta A, Pinamonti B, et al (2014) Long-term prognostic impact of therapeutic strategies in patients with idiopathic dilated cardiomyopathy: changing mortality over the last 30 years. Eur J Heart Fail 16:317- 324. https://doi.org/10.1002/ejhf.16

5. Singh P, Bhatt B , Pawar S U, et al (2018) Role of Myocardial Perfusion Study in Differentiating Ischemic versus Nonischemic Cardiomyopathy Using Quantitative Parameters. Indian Journal of Nuclear Medicine ljnm the Official Journal of the Society of Nuclear Medicine India 33(1):32-38.https://doi.org/10.4103/ijnm.IJNM_118_17

6. De Jong RM, Tio RA, Van der Harst P, et al (2009) Ischemic patterns assessed by positron emission tomography predict adverse outcome in patients with idiopathic dilated cardiomyopathy. J Nucl Cardiol 16:769-74.https://doi.org/10.1007/s12350-009-9130-9

7.Mitchell C, Rahko P S , Blauwet L A, et al (2019) Guidelines for Performing a Comprehensive Transthoracic Echocardiographic Examination in Adults: Recommendations from the American Society of Echocardiography. Journal of the American Society of Echocardiography 32( 1):164..https://doi.org/10.1016/j.echo.2018.06.004

8. Pradhan J , Senior R (2019) Assessment of myocardial viability by myocardial contrast echocardiography: current perspectives. Current Opinion in Cardiology 34(5):1. https://doi.org/10.1097/HCO.0000000000000650

9. Zhang WZ, Zha DG, Cheng GX, et al (2004) Assessment of regional myocardial blood flow with myocardial contrast echocardiography: an experimental study. Echocardiography 21: 409-416. https://doi.org/10.1111/j.0742-2822.2004.03008.x

10. Çelik T, Öztürk C, Balta S, et al (2016) Coronary microvascular dysfunction in patients with cardiac syndrome X: Ongoing debate. Int J Cardiol 218: 233-4.https://doi.org/ 10.1016/j.ijcard.2016.05.050

11. Wang X, Qiao W, Xiao Y, et al (2020) Experimental Research on the Evaluation of Left Ventricular Function by Layered Speckle Tracking in a Constrictive Pericarditis Rat Model. J Ultrasound Med. https://doi.org/10.1002/jum.15333

12. Camici PG, Amati G, Rimoldi O (2015) Coronary microvascular dysfunction: mechanisms and functional assessment. Nat Rev Cardiol. 12(1):48-62.https://doi.org/10.1038/nrcardio.2014.160

13. Mathew T, Williams L, Navaratnam G, et al (2017) Diagnosis and assessment of dilated cardiomyopathy: a guideline protocol from the British Society of Echocardiography.Echo Research and Practice 4(2):G1-G13.https://doi.org/ 10.1530/ERP-16-0037

14. Lang RM, Badano LP, Mor-Avi V, et al (2015) Recommendations for Cardiac Chamber Quantification by Echocardiography in Adults: An Update from the American Society of Echocardiography and the European Association of Cardiovascular Imaging. European Heart Journal Cardiovascular Imaging. 16(3):233-271.https://doi.org/10.1093/ehjci/jev014

15. Devereux RB, Alonso DR, Lutas EM, et al (1986) Echocardiographic assessment of left ventricular hypertrophy: comparison to necropsy findings. Am J Cardiol. 57:450-458.https://doi.org/10.1016/0002-9149(86)90771-x

16. Nagueh SF , Smiseth OA , Appleton CP, et al (2016) Recommendations for the Evaluation of Left Ventricular Diastolic Function by Echocardiography: An Update from the American Society of Echocardiography and the European Association of?Cardiovascular Imaging. Journal of the American Society of Echocardiography. 29(4):277-314.https://doi.org/10.1016/j.echo.2016.01.011

17. Roxy S, Harald B, Mark M, et al (2017) Clinical practice of contrast echocardiography: recommendation by the European Association of Cardiovascular Imaging (EACVI) 2017. eur heart j cardiovasc imaging. 18(11):1205.https://doi.org/10.1093/ehjci/jex182 
18. Pinto YM, Elliott PM, Arbustini E, et al (2016) Proposal for a revised definition of dilated cardiomyopathy, hypokinetic non-dilated cardiomyopathy, and its implications for clinical practice: a position statement of the ESC working group on myocardial and pericardial diseases. Eur Heart J. 37:1850-1858. https://doi.org/10.1093/eurheartj/ehv727

19. Chen Chunjuan, Wang Wei, Yu We (2018) Comparative analysis of risk factors for coronary heart disease, serum lipid levels, and echocardiographic features between patients with dilated cardiomyopathy and ischemic cardiomyopathy. Chinese Journal of Clinicians(Electronic Edition). https://doi.org/CNKI:SUN:ZLYD.0.2018-08-004

20. D Sanchez-Quintana, Climent V , Ho S Y (1999) Myoarchitecture and connective tissue in hearts with tricuspid atresia. Heart (British Cardiac Society) 81(2):182-191.https://doi.org/10.1136/hrt.81.2.182

21. Tanaka H, Matsumoto K, Sawa T, et al (2014) Evaluation of global circumferential strain as prognostic marker after administration of betablockers for dilated cardiomyopathy.The international journal of cardiovascular imaging 30(7):1279-1287.https://doi.org/10.1007/s10554-0140463-3

22. Briasoulis A, Mocanu M, Marinescu K, et al (2016) Longitudinal systolic strain profiles and outcomes in peripartum cardiomyopathy. Echocardiography. 33:1354-1360.https://doi.org/10.1111/echo.13277

23. Flores-Ramirez R, Azpiri-Lopez JR, Gonzalez-Gonzalez JG,et al (2017) Global longitudinal strain as a biomarker in diabetic cardiomyopathy. A comparative study with Gal-3 in patients with preserved ejection fraction. Arch Cardiol Mex 87(4):278-

285.https://doi.org/10.1016/j.acmx.2016.06.002.

24. Minoshima M, Noda A, Kobayashi M, et al (2016) Endomyocardial radial strain rate imaging during dobutamine stress echocardiography for the evaluation of contractile reserve in patients with dilated cardiomyopathy. J Clin Ultrasound. 44(9):555-

560.https://doi.org/10.1002/jcu.22376

25. Brecker SJ (2000) The importance of long axis ventricular function. Heart 84:577-579.https://doi.org/10.1136/heart.84.6.577

26. Smith SP, Secomb TW, Hong BD, et al (2016) Time-dependent regional myocardial strains in patients with heart failure with a preserved ejection fraction. Biomed Res Int 2016: 8957307https://doi.org/10.1155/2016/8957307

27.Yen HS (2009) Anatomy and myoarchitecture of the left ventricular wall in normal and in disease. Eur J

Echocardiogr.10(8).https://doi.org/10.1093/ ejechocard/jep159

28. Shi Jing, Pan Cuizhen, Shu Xianhong, et al (2015) Quantitative evaluation of left ventricular myocardial stratification strain in normal adults by two-dimensional speckle tracking imaging. Chinese Journal of Ultrasonography. 24(5):378-381.https://doi.org/10.1016/j.echo.2017.11.007

29. De Leeuw N, Ruiter DJ, Balk AH, et al (2001) Histopathologic findings in explanted heart tissue from patients with end-stage idiopathic dilated cardiomyopathy.Transpl Int 14(5):299-306.https://doi.org/10.1007/s001470 100339

30.Zheng Ying, Yan Ruiling, Chen Kundi (2016) Assessment of left ventricular systolic function in patients with dilated cardiomyopathy using speckle tracking echocardiography. Medical Journal of National Defending Forces in Northwest China 5:309-311.

31.Crea F, Camici PG, Bairey Merz CN (2014) Coronary microvascular dysfunction: an update. Eur Heart J 17:1101-11.https://doi.org/10.1093/ eurheartj/eht513

32. Sun L, Wang Z, Xu T, et al (2018) The value of real-time myocardial contrast echocardiography for detecting coronary microcirculation function in coronary artery disease patients. anatolian journal of cardiology 19(1):27.https://doi.org/ 10.14744/AnatolJCardiol.2017.8041

33. Camici PG , Amati G, Rimoldi O (2014) Coronary microvascular dysfunction: mechanisms and functional assessment. Nature Reviews Cardiology 12(1):48-62.https://doi.org/10.1038/nrcardio.2014.160

34. Chiu B (2010) Dilated cardiomyopathy etio-morphologic investigation. Frontiers in Bioscience S2(1):112-116.https://doi.org/10.2741/s50

35. Spoladore R, Fisicaro A, Faccini A, et al (2014) Coronary microvascular dysfunction in primary cardiomyopathies. Heart 100(10):806813.https://doi.org/ 10.1136/heartjnl-2013-304291

36. Zhang Yanting (2019) Relationship between myocardial strain and myocardial fibrosis in patients with end-stage DCM evaluated by 3D-STE. Huazhong University of science and technology 2019(Doctoral dissertation). 
37. Zeng Dinyin, Wang Yong (2017) Understanding and Prospects of Anatomy, Physiology and Pathology of Coronary Artery Microcirculation. Journal of Internal Medicine Concepts \& Practice 12(01):33-36.

38. Joerg Herrmann, Juan Carlos Kaski, Amir Lerman (2012) Coronary microvascular dysfunction in the clinical setting: from mystery to reality. European Heart Journal 33(22):2771-2783.https://doi.org/10.1093/eurheartj/ ehs246

39. Neglia D, L'Abbate A (2005) Coronary microvascular dysfunction and idiopathic dilated cardiomyopathy. Pharmacological Reports Pr 57 (Suppl):151-155.

40. RMD Jong, Tio R A, Harst $P$, et al (2009) Ischemic patterns assessed by positron emission tomography predict adverse outcome in patients with idiopathic dilated cardiomyopathy. Journal of Nuclear Cardiolog 16(5):769-774. https://doi.org/10.1007/s12350-009-9130-9

41. Monakier D, Woo A, Vannan MA, et al (2004) Myocardial contrast echocardiography in chronic ischemic and nonischemic cardiomyopathies. Cardiology Clinics 22(2):269-282.https://doi.org/10.1016/j.ccl.2004.03.001

42. Shi J, Pan C, Kong D, et al (2016) Left Ventricular Longitudinal and Circumferential Layer-Specific Myocardial Strains and Their Determinants in Healthy Subjects. Echocardiography 33(4):510-518.https://doi.org/10.1111/ echo.13132

\section{Tables}

Table 1 General Information and conventional echocardiography parameters of the patients 


\begin{tabular}{|c|c|c|c|c|}
\hline Parameter & $\mathrm{DCM} \otimes \mathrm{n}=24 \bigotimes$ & Control $₫ n=19 \rrbracket$ & $t / \chi^{2}$ & $P$ \\
\hline Gende $\varangle$ Female/Male $\rrbracket$ & $17 / 7$ & $13 / 6$ & 0.029 & 0.864 \\
\hline Age $\Downarrow$ year $\rrbracket$ & $44.08 \pm 8.89$ & $41.79 \pm 7.23$ & 0.933 & 0.356 \\
\hline $\mathrm{BSA} \rrbracket \mathrm{m}^{2} \square$ & $1.93 \pm 0.16$ & $1.91 \pm 0.17$ & 0.356 & 0.724 \\
\hline SBP $₫ \mathrm{mmHg} \rrbracket$ & $120.75 \pm 12.80$ & $115.89 \pm 9.79$ & 1.410 & 0.166 \\
\hline $\mathrm{DBP} \otimes \mathrm{mmHg} \otimes$ & $75.96 \pm 8.88$ & $73.32 \pm 8.47$ & 0.995 & 0.326 \\
\hline 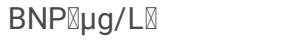 & $1950.08 \pm 836.34$ & $17.40 \pm 8.18$ & 11.320 & 0.000 \\
\hline CTnI $\nabla \mu \mathrm{g} / \mathrm{L} \bigotimes$ & $0.01(0.01,0.02)$ & $0.01(0,0.02)$ & 0.030 & 0.862 \\
\hline CRE $₫ \mu \mathrm{mol} / \mathrm{L} \nabla$ & $74.58 \pm 11.66$ & $69.89 \pm 10.32$ & 1.397 & 0.170 \\
\hline 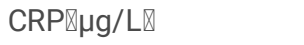 & $3.18 \pm 1.52$ & $2.89 \pm 1.25$ & 0.676 & 0.503 \\
\hline HR囚bpm® & $77.25 \pm 11.47$ & $79.21 \pm 12.45$ & 0.531 & 0.599 \\
\hline LVDd $\nabla \mathrm{mm} \rrbracket$ & $66.36 \pm 6.14$ & $47.80 \pm 3.64$ & 12.330 & 0.000 \\
\hline LVDs $₫ \mathrm{~mm} \rrbracket$ & $56.16 \pm 6.79$ & $30.30 \pm 3.55$ & 16.095 & 0.000 \\
\hline IVS』mm》 & $7.90 \pm 0.73$ & $8.34 \pm 0.60$ & -2.188 & 0.034 \\
\hline LVPW『mm® & $8.11 \pm 1.04$ & $8.24 \pm 0.81$ & -0.442 & 0.661 \\
\hline LVMI $ه g / m^{2} \square$ & $117.70 \pm 30.19$ & $69.35 \pm 10.32$ & 7.324 & 0.000 \\
\hline LVEDV『ml $\bigotimes$ & $186.58 \pm 76.29$ & $105.53 \pm 12.93$ & 5.112 & 0.000 \\
\hline LVESV『ml『 & $130.22 \pm 63.79$ & $31.17 \pm 6.35$ & 7.560 & 0.000 \\
\hline LVEF凹\%凹 & $28.88 \pm 7.91$ & $63.22 \pm 3.59$ & 18.951 & 0.000 \\
\hline FS』\%》 & $14(12.25,17.75)$ & $33.00(31.00,35.00)$ & -16.225 & 0.000 \\
\hline SV『mI $\bigotimes$ & $56.36 \pm 24.30$ & $74.36 \pm 8.18$ & -3.394 & 0.002 \\
\hline $\mathrm{CO} \otimes \mathrm{L} / \mathrm{min} \rrbracket$ & $4.26 \pm 1.64$ & $5.89 \pm 1.10$ & -3.875 & 0.001 \\
\hline $\left.\mathrm{Cl} \otimes \mathrm{L} / \triangle \mathrm{min} \cdot \mathrm{m}^{2}\right) \rrbracket$ & $2.21 \pm 0.81$ & $3.08 \pm 0.54$ & -4.228 & 0.000 \\
\hline $\mathrm{LA} \otimes \mathrm{mm} \rrbracket$ & $43.04 \pm 4.75$ & $32.21 \pm 3.33$ & 8.778 & 0.000 \\
\hline $\mathrm{LAVI} \otimes \mathrm{ml} / \mathrm{m}^{2} \square$ & $33.92(29.90,49.70)$ & $26.42(23.92,29.62)$ & 15.534 & 0.000 \\
\hline E peak $\ \mathrm{~cm} / \mathrm{s} \rrbracket$ & $78.03 \pm 22.27$ & $87.59 \pm 13.13$ & -1.753 & 0.088 \\
\hline A peak $\ \mathrm{~cm} / \mathrm{s} \rrbracket$ & $52.48 \pm 26.60$ & $68.98 \pm 21.15$ & -2.266 & 0.029 \\
\hline$E / A$ & $1.99 \pm 1.22$ & $1.38 \pm 0.48$ & 2.239 & 0.032 \\
\hline Septal $\mathrm{e}^{\square} \nabla \mathrm{cm} / \mathrm{s} \nabla$ & $4.99(4.23,7.24)$ & $8.32(7.70,10.10)$ & 16.651 & 0.000 \\
\hline Septal $a^{\square} \llbracket \mathrm{cm} / \mathrm{s} \rrbracket$ & $5.86 \pm 2.29$ & $10.28 \pm 3.79$ & -4.481 & 0.000 \\
\hline Lateral $\mathrm{e}^{\square} \rrbracket \mathrm{cm} / \mathrm{s} \rrbracket$ & $5.92(4.92,7.84)$ & $12.90(10.80,15.10)$ & 21.975 & 0.000 \\
\hline Lateral $\mathrm{a}^{\square} \rrbracket \mathrm{cm} / \mathrm{s} \rrbracket$ & $6.87 \pm 2.90$ & $11.95 \pm 3.98$ & -4.670 & 0.000 \\
\hline Average $\mathrm{E} / \mathrm{e}^{\square}$ & $14.55 \pm 6.27$ & $8.60 \pm 2.26$ & 4.311 & 0.000 \\
\hline
\end{tabular}

Data are mean \pm SD or median (Q25-Q75). BNP: brain natriuretic peptide; CTnl: cardiac troponin l; CRE: creatinine; CRP: c-reactive protein; HR: heart rate.

Table 2 Comparison of parameters of stratified strain and perfusion between two groups 


\begin{tabular}{|c|c|c|c|c|}
\hline Parameter & DCM & Control & $t / Z \square$ & $P$ \\
\hline LSendo®\%区 & $-9.80(-13.68,-6.60)$ & $-20.65(-27.03,-14.88)$ & 216.928 & 0.000 \\
\hline LSmid $\otimes \% \bigotimes$ & $-7.65(-11.30,-4.80)^{\star}$ & $-17.40(-22 \cdot 80,-12 \cdot 30)^{\star}$ & 220.989 & 0.000 \\
\hline LSepi®\%区 & $-6.00(-10.08,-1.48)^{\star} \triangle$ & $-13.75(-20.03,-8.20)^{\star} \triangle$ & 130.612 & 0.000 \\
\hline CSendo $\otimes \% \square$ & $-12.30(-18.50,-7.63)$ & $-33.75(-41.90,-26.00)$ & 381.415 & 0.000 \\
\hline CSmid $\approx \% \bigotimes$ & $-8.40(-13.88,-4.20)^{\star}$ & $-23.50(-30.85,-17.38)^{\star}$ & 251.909 & 0.000 \\
\hline CSepiष\%区 & $-5.55(-10.18,3.98) \star \triangle$ & $-15.55(-23.20,-10.03) \star \Delta$ & 173.306 & 0.000 \\
\hline Aendo $\triangle \mathrm{dB} \triangle$ & $20.55(17.29,24.21)$ & $28.35(23.43,33.34)$ & 144.174 & 0.000 \\
\hline Amid $\rrbracket \mathrm{dB} \rrbracket$ & $18.61(15.24,22.05)^{\star}$ & $25.69(20.72,30.74)^{\star}$ & 127.327 & 0.000 \\
\hline Aepi $₫ \mathrm{~dB} \rrbracket$ & $18.07(14.32,22.14)^{\star}$ & $24.91(19.43,29.86)^{\star}$ & 102.548 & 0.000 \\
\hline Bendo $₫ s^{-1} \square$ & $6.69(3.02,13.73)$ & $7.70(4.07,15.47)$ & 2.093 & 0.148 \\
\hline$\beta \operatorname{mid} \llbracket s^{-1} \square$ & $5.10(1.85,10.64)^{\star}$ & $7.64(3.72,15.49)^{\star}$ & 25.637 & 0.000 \\
\hline 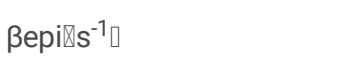 & $4.65(2.35,11.32)^{\star}$ & $7.51(4.61,18.86)^{\star}$ & 15.257 & 0.000 \\
\hline$A \times \beta e n d o \rrbracket d B \times s^{-1} \square$ & $139.44(53.85,288.16)$ & $229.20(118.58,540.66)$ & 30.220 & 0.000 \\
\hline$A \times \beta m i d \rrbracket d B \times s^{-1} \square$ & $89.64(36.16,210.22)^{*}$ & $202.46(101.46,392.02)^{\star}$ & 54.434 & 0.000 \\
\hline$A \times \beta e p i 囚 d B \times s^{-1} \square$ & $87.70(30.80,200.92)^{\star}$ & $182.78(86.02,354.75)^{\star}$ & 36.917 & 0.000 \\
\hline Time to peak endo (sec) & $4.57(3.97,5.04 \rrbracket$ & $2.73(2.08,3.33)$ & -21.167 & 0.000 \\
\hline Time to peak mid (sec) & $4.63(4.12,5.06)$ & $2.71(2.13,3.21)$ & -21.703 & 0.000 \\
\hline Time to peak epi (sec) & $4.58(4.02,4.99)$ & $2.61(2.04,3.20)$ & -21.463 & 0.000 \\
\hline Cardiac cycle endo & $6.00(5.03,7.02)$ & $3.51(2.80,4.34)$ & -18.95 & 0.000 \\
\hline Cardiac cycle mid & $6.13(5.28,6.98)$ & $3.48(2.84,4.25)$ & -19.59 & 0.000 \\
\hline Cardiac cycle epi & $6.07(5.09,6.90)$ & $3.35(2.69,4.21)$ & -19.48 & 0.000 \\
\hline
\end{tabular}

Data are median (Q25-Q75). endo: endocardium; mid:middle layer; epi: epicardium.

* : There was statistical significance compared with endocardium $(P<0.05) ; \triangle$ : There was statistically significant compared with middle layer $(P<0.05)$.

Table 3 Correlation between layered strain and layered perfusion $(r)$

\begin{tabular}{|c|c|c|c|c|c|c|c|c|c|c|c|}
\hline Parameter & Aendo & Bendo & A $\times$ Bendo & Parameter & Amid & $\beta$ mid & $A \times \beta$ mid & Parameter & Aepi & ßepi & A×ßері \\
\hline CSendo & $-0.383^{* *}$ & $-0.084 *$ & $-0.140 * \star$ & CSmid & $-0.255^{\star \star}$ & -0.060 & $-0.080 *$ & CSepi & $-0.208^{\star \star}$ & -0.049 & $-0.081 *$ \\
\hline LSendo & $-0.500 * *$ & $-0.100 * *$ & -0.151 ** & LSmid & $-0.279 * \star$ & -0.064 & $-0.101 * *$ & LSepi & $-0.190 * *$ & $-0.102^{\star \star}$ & $-0.118 * *$ \\
\hline
\end{tabular}

* indicates $P<0.05 ;$ ** $P \otimes 0.01$ 


\section{Figures}

A

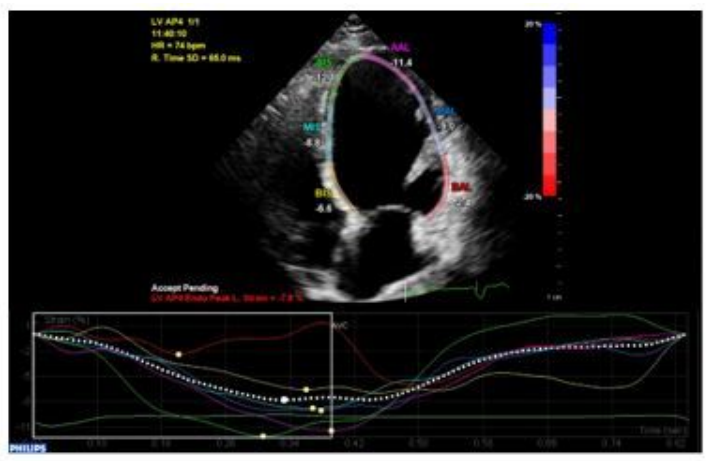

B
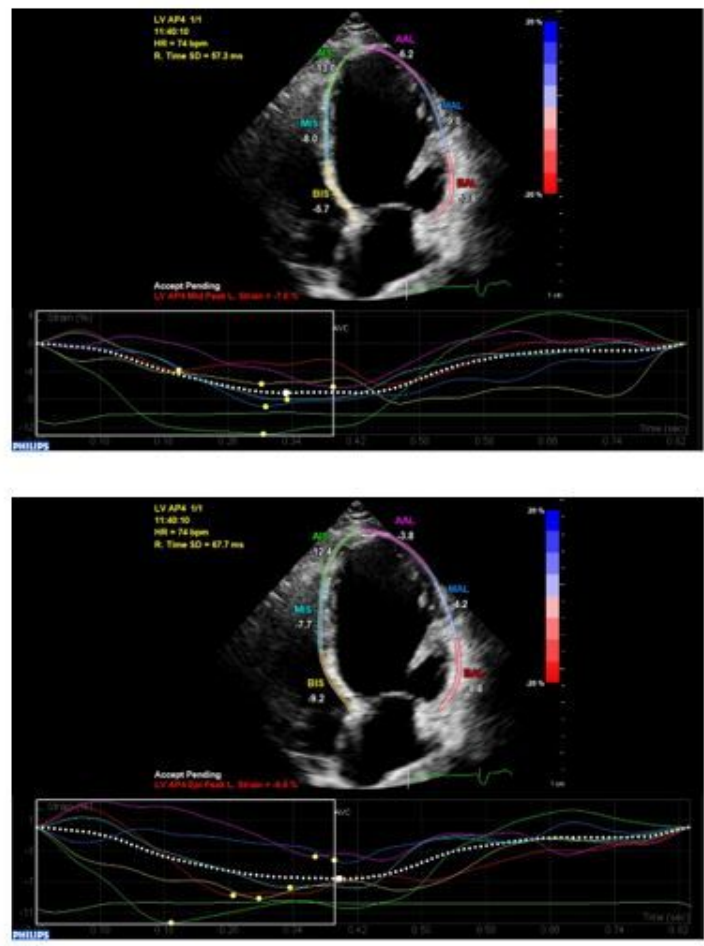

Figure 1

The calculation of the GLS for left ventricular endocardium (A)囚middle layer (B) and epicardium (C) in patients with DCM囚apical 4-chamber》 


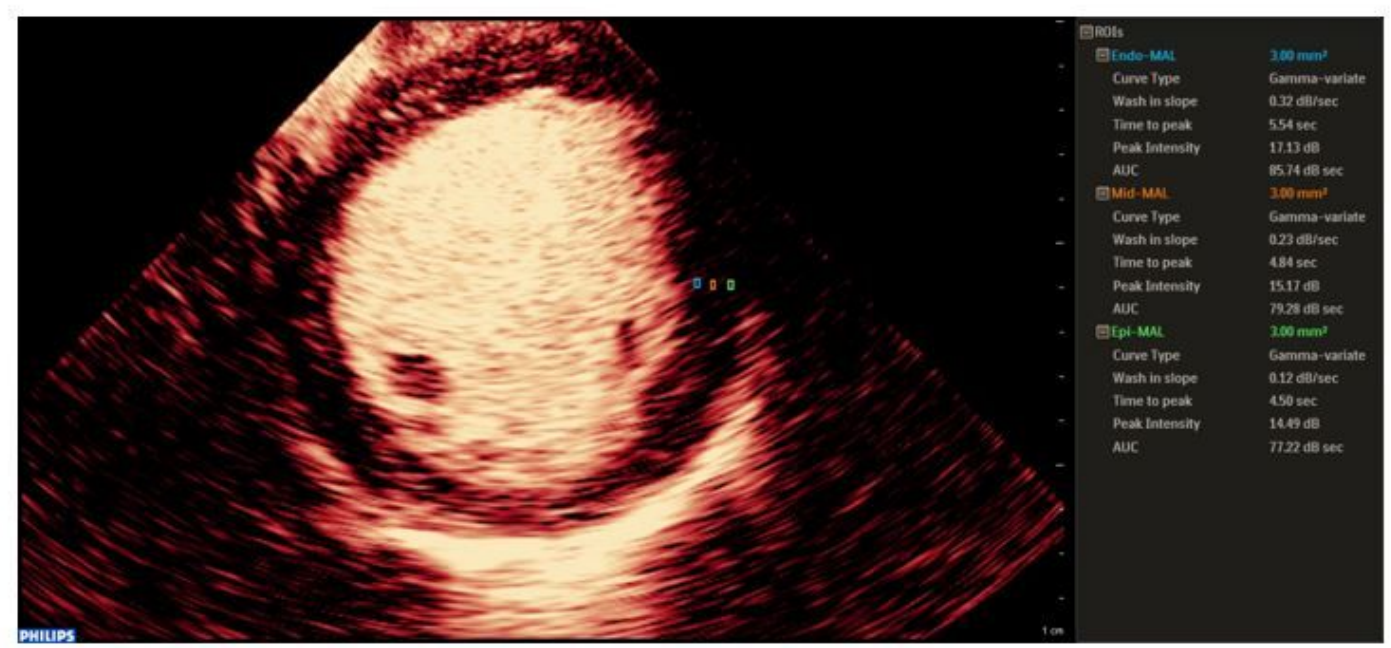

Figure 2

The region of interest (ROIs) and results of myocardial perfusion imaging of left ventricular three-layer myocardium in patients with with DCM (left ventricular short axis section)
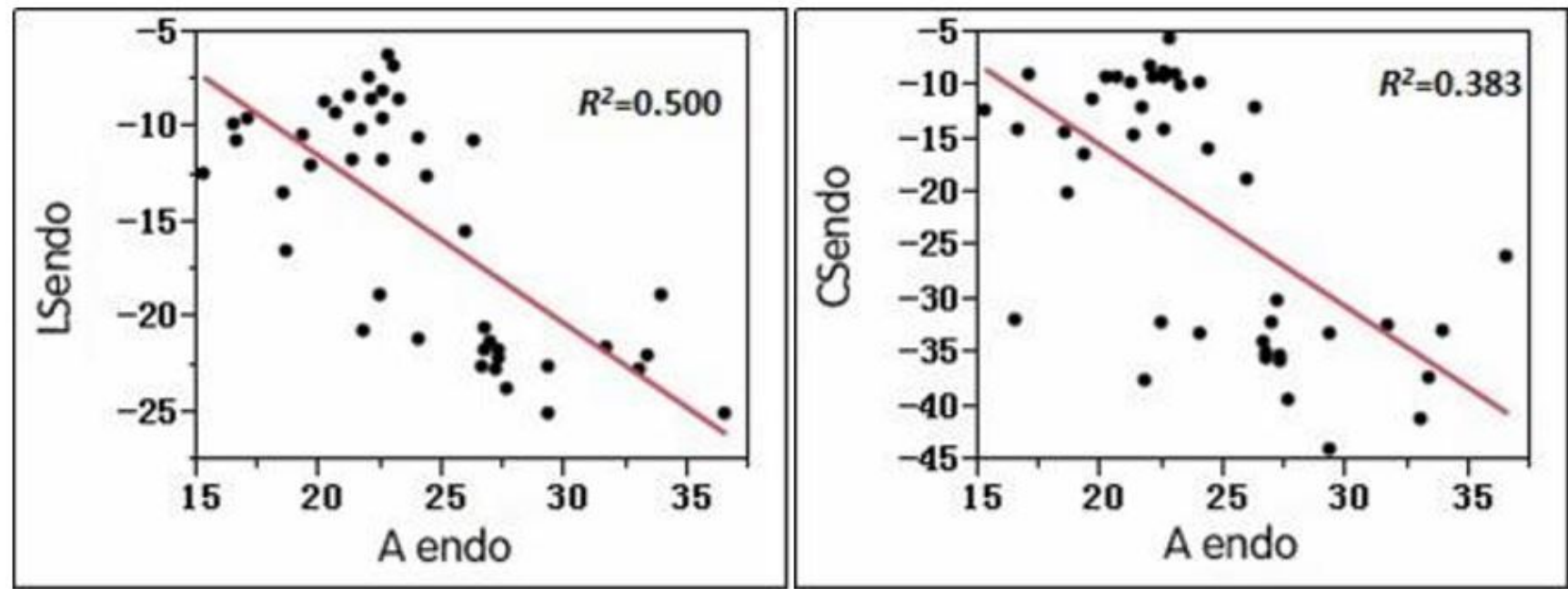

Figure 3

The correlation between LSendo, CSendo and A endo 